\title{
INFLUÊNCIA DO AMBIENTE SOBRE A FUNCIONALIDADE DE CRIANÇAS
}

\section{Influence of the environment on children's functionality \\ Influencia del ambiente para la funcionalidad de niños}

\author{
Dayse Danielle de Oliveira Silva (iD \\ Universidade do Estado do Pará - UEPA - Belém (PA) - Brasil
}

Adrielly Elane Sousa Maia iD

Universidade do Estado do Pará - UEPA - Belém (PA) - Brasil

Ana Paula Monteiro de Araújo (iD

Centro Universitário do Estado do Pará - CESUPA - Belém (PA) - Brasil

Hannah Nazareth Muribeca Athar (iD

Universidade da Amazônia - UNAMA - Belém (PA) - Brasil

Paula Cristina Soares Mesquita (iD

Faculdade de Medicina da Universidade de São Paulo - FMUSP - São Paulo (SP) - Brasil

Juliana Maciel de Queiroz (D)

Universidade do Estado do Pará - UEPA - Belém (PA) - Brasil

\section{RESUMO}

Objetivo: Avaliar o perfil funcional de crianças ribeirinhas a fim de correlacionar a influência do ambiente no desempenho das suas atividades relacionadas ao autocuidado, à mobilidade e à função social. Métodos: Estudo descritivo e transversal realizado no município de Cachoeira do Arari, Pará, comunidade Caracará, em maio de 2017, com 50 crianças, de 5 anos a 7 anos e 6 meses, e seus respectivos cuidadores. Aplicou-se um questionário de identificação infantil como critério de inclusão e avaliaramse os participantes selecionados por meio do Inventário de Avaliação Pediátrica de Incapacidades (PEDI) e pela entrevista sociodemográfica (ABEP). Submeteram-se os dados ao teste de aderência de Shapiro-Wilk e, em seguida, aos testes do quiquadrado e de correlação de Pearson, com nível de significância de $5 \%$. Resultados: As crianças apresentaram desempenho funcional adequado para as áreas: Habilidades funcionais em autocuidado (HFAC) $(70 \%, p<0,001)$; Habilidades funcionais em mobilidade (HFM) $(72 \%, p<0,001)$; Habilidades funcionais em função social (HFFS) $(94 \%, p<0,001)$; Assistência do cuidador em autocuidado (ACAC) (94\%, p<0,001); Assistência do cuidador em função social (ACFS) $(66 \%, p<0,001)$, exceto na Assistência do cuidador em mobilidade (ACM). Uma correlação moderadamente positiva foi encontrada entre as áreas: HFFS e HFAC ( $r=0,618$, $p<0,001)$, ACAC e HFAC ( $r=0,641, p<0,001)$, ACM e HFM $(r=0,673, p<0,001)$, ACFS e HFAC $(r=0,581, p<0,001)$ e ACFS e HFFS $(0,647, p<0,001)$. Conclusão: Observou-se que as crianças ribeirinhas apresentam perfil funcional adequado à faixa etária e que o ambiente não influenciou negativamente nas suas atividades relacionadas ao autocuidado, à mobilidade e à função social.

Descritores: Criança; Fisioterapia; Características Culturais.

\section{ABSTRACT}

Objective: To evaluate the functional profile of riverside children to correlate the influence of the environment on the performance of their activities related to self-care, mobility, and social function. Methods: A descriptive and cross-sectional study conducted in the municipality of Cachoeira do Arari, Pará, Caracará community, in May 2017, with 50 children, aged from 5 years to 7 years and 6 months, and their respective caregivers. A child identification questionnaire was applied as an inclusion criterion and the selected participants were assessed through the Pediatric Disability Assessment Inventory (PEDI) and the sociodemographic interview $(A B E P)$. The data were submitted to the Shapiro-Wilk adherence test and then to the chi-square and Pearson correlation tests, with a significance level of $5 \%$. Results: The children showed adequate functional performance for the following areas: Functional skills in self-care (HFAC) (70\%, $p<0.001)$; Functional mobility skills (HFM) $(72 \%, p<0.001)$; Functional skills in social function (HFFS) (94\%, $p<0.001)$; Caregiver assistance in self-care (ACAC) (94\%, $p<0.001)$; and Caregiver assistance in social function (ACFS) $(66 \%, p<0.001)$, except for Mobile caregiver assistance (ACM). A moderately positive correlation was found

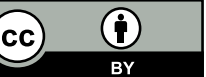


between the areas: HFFS and HFAC ( $r=0.618, p<0.001), A C A C$ and HFAC $(r=0.641, p<0.001), A C M$ and HFM ( $r=0.673$, $p$ <0.001), ACFS and HFAC ( $r=0.581, p<0.001)$, and ACFS and HFFS (0.647, $p$ <0.001). Conclusion: It was observed that riverside children have a functional profile appropriate to the age group and that the environment did not negatively influence their activities related to self-care, mobility, and social function.

Descriptors: Child; Physiotherapy; Cultural Characteristics.

\section{RESUMEN}

Objetivo: Evaluar el perfil funcional de niños ribereños para correlacionar la influencia del ambiente para el rendimiento de sus actividades relacionadas con el auto cuidado, la movilidad y la función social. Métodos: Estudio descriptivo y transversal realizado en el municipio de Cachoeira de Arari, Pará, comunidad Caracará, en mayo de 2017 con 50 niños entre 5 y 7 años y 6 meses y sus respectivos cuidadores. Se aplicó un cuestionario para la identificación infantil como criterio de inclusión y se evaluaron los participantes elegidos a través del Inventario de Evaluación Pediátrica de Discapacidades (IEPD) y por la entrevista sociodemográfica $(A B E P)$. Se ha puesto los datos a la prueba de adherencia de Shapiro-Wilk y, a continuación, a las pruebas de Chi-cuadrado y correlación de Pearson con el nivel de significancia del 5\%. Resultados: Los niños presentaron el rendimiento funcional adecuado para las áreas: Habilidades funcionales en el auto cuidado (HFAC) (70\%, $p<0,001)$; Habilidades funcionales en la movilidad (HFM) (72\%, p<0,001); Habilidades funcionales en la función social (HFFS) $(94 \%, p<0,001)$; Asistencia del cuidador para el auto cuidado (ACAC) (94\%, $p<0,001)$; Asistencia del cuidador para la función social (ACFS) (66\%, p<0,001), excepto en la Asistencia del cuidador en la movilidad (ACM). Ha sido encontrada una correlación positiva moderada entre las áreas: HFFS y HFAC ( $r=0,618, p<0,001), A C A C$ y HFAC $(r=0,641, p<0,001), A C M$ y HFM $(r=0,673, p<0,001)$, ACFS y HFAC $(r=0,581, p<0,001)$ y ACFS y HFFS $(0,647, p<0,001)$. Conclusión: Se observó que los niños ribereños presentan el perfil funcional adecuado para su franja de edad y que el ambiente no ha influenciado de manera negativa con sus actividades relacionadas con el auto cuidado, la movilidad y la función social.

Descriptores: Niño; Fisioterapia; Características Culturales.

\section{INTRODUÇÃO}

A promoção da saúde consiste em um conjunto de estratégias, no âmbito individual e coletivo, rastreando e problematizando os fatores determinantes das condições de saúde e doença como forma de produzir saúde. Esses fatores podem ser analisados no espaço escolar, por meio de uma política intersetorial do Ministério da Saúde e do Ministério da Educação, o Programa Saúde na Escola, instituído em 2007, que prevê ações que variam de acordo com o nível de ensino e compreendem a avaliação das condições de saúde; a promoção da saúde e prevenção das doenças e agravos; e a capacitação permanente dos profissionais de saúde e educação. Uma ação bastante comum é a avaliação do desenvolvimento integral da criança e seus fatores de risco para atraso(1,2).

O desenvolvimento neurológico durante a infância depende da saúde física e da disposição genética, e progride sob a influência do contexto social e do ambiente ${ }^{(3)}$. Para realizar atividades no dia a dia, são necessárias habilidades motoras e de interação social. O desenvolvimento motor baseia-se em ações que uma pessoa aprova ao interagir e mover objetos de tarefa e a si próprios em torno de um ambiente de tarefa ${ }^{(4,5)}$.

Crianças em áreas urbanas frequentemente vivem em melhores condições do que crianças em áreas rurais, graças a padrões mais altos de saúde, proteção, educação e saneamento. No entanto, os avanços urbanos têm sido desiguais, e milhões de crianças em contextos urbanos marginalizados enfrentam diariamente desafios e privações de seus direitos ${ }^{(6)}$.

Diferente do contexto urbano, as crianças ribeirinhas convivem constantemente com a presença da mata e dos rios, e suas atividades estão relacionadas àquelas desenvolvidas pelos adultos, especialmente por seus pais. Elas participam de todos os momentos da vida da comunidade, no trabalho, em casa, no lazer e nas atividades religiosas. As brincadeiras observadas com mais frequência na comunidade ribeirinha foram as de faz de conta ou jogo de papéis, seguidas de outros tipos de brincadeiras, como nadar no rio, empinar pipa/papagaio, jogar bola, subir em árvores, correr nos terreiros e nas pontes, procurar objetos nas matas e passear de canoa ${ }^{(7)}$.

Em meio a biodiversidade e cultura, com diversas peculiaridades, como o modo de vida extrativista, as comunidades ribeirinhas sofrem com a ausência de energia elétrica, falta de saneamento básico e, principalmente, com o precário acesso às políticas públicas nas áreas de educação e saúde ${ }^{(8)}$. Entre os fatores ambientais, o lar e a família desempenham um papel primordial durante os primeiros anos da infância. A educação dos pais, o status socioeconômico, o tamanho da família e a interação com os irmãos são os principais elementos do ambiente próximo da criança ${ }^{(9,10)}$. 
Em um cenário comum entre os municípios com população ribeirinha, segundo o Programa das Nações Unidas para o Desenvolvimento(11), o município de Cachoeira do Arari, Pará, se encontra entre os piores no Índice de Desenvolvimento Humano Municipal $(0,546)$, o que sugere que seus habitantes têm poucas oportunidades sociais de desenvolvimento, podendo estar em desvantagem no que se refere à sua participação e inclusão social.

Devido às condições socioeconômicas e estruturais, o presente estudo tem como objetivo avaliar o perfil funcional de crianças ribeirinhas, a fim de correlacionar a influência do meio ambiente no desempenho das suas atividades relacionadas ao autocuidado, à mobilidade e à função social.

\section{MÉTODOS}

Trata-se de um estudo descritivo, de caráter transversal e exploratório, de natureza quantitativa, realizado no município de Cachoeira do Arari, na comunidade Caracará, Pará, em maio de 2017.

Participaram do estudo crianças devidamente matriculadas em escolas municipais e seus respectivos cuidadores. Considerando uma população local de 97 crianças registradas e que se adequavam aos critérios de inclusão, foi calculada a amostra probabilística obtendo-se $n=50(51,55 \%)$ crianças, proporção que variou no intervalo de confiança (95\%) entre $33,4 \%$ e $53,2 \%$.

Como critérios de inclusão, considerou-se crianças entre 5 anos e 7 anos e 6 meses, de ambos os sexos, cujos cuidadores se disponibilizassem a responder aos instrumentos de avaliação com informações referentes à funcionalidade e ao perfil socioeconômico das famílias. Já como critério de exclusão, considerou-se as crianças que apresentaram qualquer risco biológico (intercorrências nos períodos pré, peri e pós-natal, como prematuridade, baixo peso ao nascer, descolamento da placenta, sangramentos vaginais, asfixia perinatal, distúrbios bioquímicos e hematológicos, microcefalia, malformações e infecções congênitas), com distúrbios associados (como retardo mental, problemas neurológicos ou ortopédicos, e presença de distúrbios sensoriais, visuais ou auditivos) e/ou sinais de padrões anormais do desenvolvimento neuromotor identificados no questionário previamente respondido pelos responsáveis/cuidadores da criança.

A coleta de dados aconteceu nas escolas que as crianças frequentavam, no horário de maior conveniência para o cuidador, em lugar agradável para estimular a participação do entrevistado e após assinatura do Termo de Consentimento Livre e Esclarecido e do Termo de Assentimento da Criança.

Utilizaram-se os seguintes instrumentos avaliativos para a coleta de dados: o Inventario de Avaliação Pediátrica de Incapacidades (PEDI), versão brasileira validada em $2005^{(10)}$, aplicado por meio de entrevista estruturada com os responsáveis que passam mais tempo com a criança, com o objetivo de descrever, detalhadamente, o desempenho funcional da criança. A versão foi traduzida e adaptada do original norte-americano Pediatric Evaluation of Disability Inventory seguindo todos os critérios e procedimentos descritos na literatura, que incluíram as etapas de tradução, adaptação cultural e desenvolvimento de normas brasileiras, sendo realizado pelos Departamentos de Terapia Ocupacional e de Fisioterapia da Universidade Federal de Minas Gerais. Seus dados normativos, que constituíram a amostra de padronização brasileira, foram obtidos através de 276 crianças da Região Metropolitana de Belo Horizonte ${ }^{(10)}$.

O PEDI é constituído pelas áreas de autocuidado, mobilidade e função social, que são compostas por três partes: I - Habilidades funcionais, que mede a capacidade da criança de desempenhar determinadas habilidades funcionais; II - Assistência do cuidador, que avalia a quantidade habitual de ajuda fornecida durante a realização de atividades funcionais básicas e III - Modificação do ambiente, que identifica o número de atividades funcionais para as quais são necessárias modificações ${ }^{(10)}$.

Utilizou-se, também, a entrevista sociodemográfica, com o intuito de fornecer dados sobre características individuais da mãe e do cônjuge (estado civil, religião etc.), da composição familiar e demográfica (tipo de família, número de filhos e ordem de nascimento, número de pessoas residentes, número de cômodos etc.), e dados sobre escolaridade, renda e ocupação remunerada dos pais, adaptada do roteiro da Associação Brasileira de Empresas de Pesquisa (ABEP), intitulado Critério de Classificação Econômica Brasil(12).

A fim de padronizar a aplicação dos instrumentos e mensurar a confiabilidade da coleta de dados, aplicou-se previamente o coeficiente de correlação intraclasse (ICC), obtendo-se a replicabilidade considerada excelente $(I C C>0,90, p<0,05)$.

Os dados coletados foram submetidos ao teste de aderência de Shapiro-Wilk e, em seguida, aos testes do qui-quadrado e de correlação de Pearson, com nível de significância previamente adotado de $5 \%(p<0,05)$. Para a análise, utilizou-se o Statistic Package for Social Sciences (SPSS), versão 24.0. 
O presente estudo foi aprovado pelo Comitê de Ética em Pesquisa da Universidade da Amazônia - UNAMA, com o Parecer n. ${ }^{\circ}$ 2.017.531.

\section{RESULTADOS}

Participaram da pesquisa 50 crianças e seus respectivos cuidadores principais. Verificam-se, na Tabela I, as características sociodemográficas das crianças. Destas, $52 \%(n=26)$ eram do sexo masculino, $54 \%(n=27)$ possuíam 6 anos de idade e $44 \%(n=22)$ se encontravam no período escolar infantil 3.

A Tabela II caracteriza os cuidadores. Destes, $50 \%(n=25)$ eram mães da criança; $46 \%(n=23)$ avós e $4 \%$ $(n=2)$ pais. Além disso, $70 \%(n=35)$ faziam parte de uma formação familiar nuclear, $70 \%(n=35)$ possuíam o ensino fundamental incompleto e $72 \%(n=36)$ das mães eram pescadoras.

As participantes do estudo afirmaram que a gestação da criança não foi planejada em $56 \%(n=28)$ dos casos, sendo que $28 \%(n=14)$ das crianças nasceram da primeira gestação e $34 \%(n=17)$ das mães estavam na $5^{a}$ gestação ou mais.

Entre as famílias, $62 \%(n=31)$ receberam classificação na classe $D$, seguindo a classificação da $A B E P(10)$, que distingue os grupos de acordo com a posse de itens de bens de consumo doméstico.

Tabela I - Características sociodemográficas das crianças (n=50). Cachoeira do Arari, Pará, 2017.

\begin{tabular}{lccc}
\hline Caracterização da amostra - crianças & $\mathbf{n}$ & $\%$ & $\mathbf{p}^{(1)}$ \\
\hline Sexo da criança & & & \\
$\quad$ Feminino & 24 & 48,00 & 0,777 \\
$\quad$ Masculino & 26 & 52,00 & \\
Idade da criança & & & \\
5 anos & 15 & 30,00 & $0,004^{* *}$ \\
6 anos & 27 & 54,00 & \\
7 anos & 8 & 16,00 & \\
Escolaridade da criança & & & \\
$1^{\circ}$ Ano & 19 & 38,00 & $0,062^{*}$ \\
$2^{\circ}$ Ano & 9 & 18,00 & \\
Infantil 3 & 22 & 44,00 & \\
\hline
\end{tabular}

(1): Teste qui-quadrado de Pearson para tendência $(p<0,05)$; *: valores significativos; **: valores altamente significativos

No presente estudo, as crianças apresentaram desempenho funcional adequado em todas as áreas da PEDI $(p<0,05)$, exceto para a Assistência do cuidador em mobilidade, em que as proporções foram equivalentes, com $56 \%$ $(n=28)$ das crianças com desempenho adequado e $44 \%(n=22)$ das crianças com desempenho atrasado. No caso do desenvolvimento atrasado, observa-se que a assistência do cuidador para mobilidade apresentou um número relevante de crianças $(44 \%, n=22)$, conforme a Tabela III.

$\mathrm{Na}$ Tabela IV, observa-se que as crianças apresentaram desempenho adequado (escore entre 30 e 70) nas Habilidades funcionais em função social $(\mu=46.32)$, e na Assistência do cuidador em autocuidado $(\mu=45.89)$ e Função Social $(\mu=48.00)$ o desempenho das crianças foi melhor do que nas outras habilidades e assistências, dentro do intervalo de normalidade.

A Tabela $V$ apresenta que a área de Autocuidado (HFAC) obteve associação positiva com as Habilidades funcionais em mobilidade (HFM) e Função Social (HFFS), indicando que, quanto maior a funcionalidade nas atividades sociais e motoras, maior a capacidade da criança na realização de tarefas do autocuidado, tais como: vestir-se, alimentarse e realizar sua higiene pessoal.

Verificou-se, também, associação positiva entre HFAC e ACAC $(r=0,641 ; p<0,05)$ e mobilidade $(A C M)(r=$ 0,$500 ; p=0,000$ ), indicando que, quanto maior for o resultado na HFAC, maior será o escore de ACAC e ACM, consequentemente, maior será o nível de independência da criança nessas áreas.

A HFM apresenta associação significativa, de moderada magnitude, com a ACM $(r=0,673 ; p=0,000)$, indicando que, quanto maior a capacidade da criança nas atividades funcionais de mobilidade, como transferências e locomoção em ambientes, menor é a quantidade de ajuda fornecida pelo cuidador e maior será sua independência quanto à mobilidade. 
Tabela II - Características sociodemográficas dos cuidadores ( $n=50)$. Cachoeira do Arari, Pará, 2017.

\begin{tabular}{|c|c|c|c|}
\hline Caracterização da amostra - cuidadores & $\mathbf{n}$ & $\%$ & $\mathbf{p}^{(1)}$ \\
\hline \multicolumn{4}{|l|}{ Tipo de família } \\
\hline Mononuclear & 4 & 8,00 & \multirow{3}{*}{$0,000^{* *}$} \\
\hline Nuclear & 35 & 70,00 & \\
\hline Nuclear estendida & 11 & 22,00 & \\
\hline \multicolumn{4}{|l|}{ Escolaridade da mãe } \\
\hline Ensino Fundamental Incompleto & 35 & 70,00 & \multirow{6}{*}{$0,000^{* *}$} \\
\hline Ensino fundamental completo & 2 & 4,00 & \\
\hline Ensino médio incompleto & 7 & 14,00 & \\
\hline Ensino médio completo & 4 & 8,00 & \\
\hline Ensino superior incompleto & 1 & 2,00 & \\
\hline Não sabe & 1 & 2,00 & \\
\hline \multicolumn{4}{|l|}{ Profissão da mãe } \\
\hline Pescadora & 36 & 72,00 & \multirow{6}{*}{$0,000^{* *}$} \\
\hline Dona de casa & 8 & 16,00 & \\
\hline Servente & 3 & 6,00 & \\
\hline Funcionária pública & 1 & 2,00 & \\
\hline Autônoma & 1 & 2,00 & \\
\hline Estudante & 1 & 2,00 & \\
\hline \multicolumn{4}{|l|}{ Ordem de nascimento da criança } \\
\hline 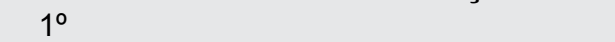 & 14 & 28,00 & \multirow{8}{*}{$0,000^{* *}$} \\
\hline $2^{\circ}$ & 10 & 20,00 & \\
\hline $3^{\circ}$ & 7 & 14,00 & \\
\hline $4^{\circ}$ & 10 & 20,00 & \\
\hline $5^{\circ}$ & 5 & 10,00 & \\
\hline $6^{\circ}$ & 2 & 4,00 & \\
\hline $7^{\circ}$ & 1 & 2,00 & \\
\hline $13^{\circ}$ & 1 & 2,00 & \\
\hline \multicolumn{4}{|l|}{ A gestação foi planejada? } \\
\hline Sim & 22 & 44,00 & \multirow{2}{*}{$0,001^{* *}$} \\
\hline Não & 28 & 56,00 & \\
\hline \multicolumn{4}{|l|}{ Número de gestações } \\
\hline 1 & 2 & 4,00 & \multirow{5}{*}{$0,011^{*}$} \\
\hline 2 & 12 & 24,00 & \\
\hline 3 & 12 & 24,00 & \\
\hline 4 & 7 & 14,00 & \\
\hline 5 ou mais & 17 & 34,00 & \\
\hline \multicolumn{4}{|l|}{ Classificação social } \\
\hline $\mathrm{C} 1$ & 2 & 4,00 & \multirow{4}{*}{$0,000^{* *}$} \\
\hline $\mathrm{C} 2$ & 10 & 20,00 & \\
\hline$D$ & 31 & 62,00 & \\
\hline $\mathrm{E}$ & 7 & 14,00 & \\
\hline
\end{tabular}

(1): Teste qui-quadrado de Pearson para tendência $(p<0,05)$; *: valores significativos; **: valores altamente significativos

Tabela III - Classificação do desempenho das crianças nas áreas avaliadas pelo escore normativo do Inventario de Avaliação Pediátrica de Incapacidades - PEDI (n=50). Cachoeira do Arari, Pará, 2017.

\begin{tabular}{|c|c|c|c|c|c|c|c|}
\hline \multirow{3}{*}{ PEDI } & \multicolumn{6}{|c|}{ Desempenho } & \multirow{3}{*}{$\mathbf{p}^{(1)}$} \\
\hline & \multicolumn{2}{|c|}{ Adequado } & \multicolumn{2}{|c|}{ Atrasado } & \multicolumn{2}{|c|}{ Adiantado } & \\
\hline & $n$ & $\%$ & $\mathrm{n}$ & $\%$ & $n$ & $\%$ & \\
\hline HFAC & 35 & 70,0 & 15 & 30,0 & 0 & 0,0 & $<0,0005^{\star *}$ \\
\hline HFM & 36 & 72,0 & 14 & 28,0 & 0 & 0,0 & $0,0002^{* *}$ \\
\hline HFFS & 47 & 94,0 & 0 & 0,0 & 3 & 6,0 & $<0,0001^{\text {** }}$ \\
\hline ACAC & 47 & 94,0 & 3 & 6,0 & 0 & 0,0 & $<0,0001^{* *}$ \\
\hline ACM & 28 & 56,0 & 22 & 44,0 & 0 & 0,0 & 0,396 \\
\hline ACFS & 33 & 66,0 & 9 & 18,0 & 8 & 16,0 & $<0,0001^{* *}$ \\
\hline
\end{tabular}

HFAC: Habilidades Funcionais em Autocuidado; HFM: Habilidades Funcionais em Mobilidade; HFFS: Habilidades Funcionais em Função Social; ACAC: Assistência do Cuidador em Autocuidado; ACM: Assistência do Cuidador em Mobilidade; ACFS: Assistência do Cuidador em Função Social

(1): Teste qui-quadrado de Pearson para tendência $(p<0,05)$; *: valores significativos; **: valores altamente significativos 
Tabela IV - Desempenho das crianças nas áreas avaliadas pelo escore normativo do Inventario de Avaliação

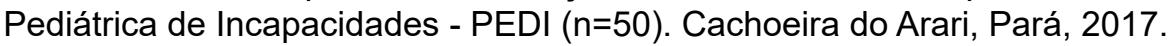

\begin{tabular}{ccccc}
\hline Variável & Média & \pm DP & Mínimo & Máximo \\
\hline HFAC & 37.13 & 12.71 & Abaixo de 10 & 69.80 \\
HFM & 38.31 & 14.43 & Abaixo de 10 & 58.10 \\
HFFS & 46.32 & 10.18 & 34.60 & 78.10 \\
ACAC & 45.89 & 9.47 & 16.30 & 62.50 \\
ACM & 34.80 & 14.54 & Abaixo de 10 & 58.40 \\
ACFS & 48.00 & 22.19 & Abaixo de 10 & 90.00 \\
\hline
\end{tabular}

HFAC: Habilidades Funcionais em Autocuidado; HFM: Habilidades Funcionais em Mobilidade; HFFS: Habilidades Funcionais em Função Social; ACAC: Assistência do Cuidador em Autocuidado; ACM: Assistência do Cuidador em Mobilidade; ACFS: Assistência do Cuidador em Função Social; DP: desvio padrão

Tabela V - Correlação entre Habilidades funcionais e Assistência do cuidador $(n=50)$. Cachoeira do Arari, Pará, 2017.

\begin{tabular}{lccc}
\hline \multicolumn{2}{c}{ Variáveis correlacionadas } & Coeficiente de correlação $(\mathbf{r})$ & $\mathbf{p}$ \\
\hline HFM & HFAC & 0,464 & 0,002 \\
HFFS & HFAC & 0,618 & 0,000 \\
HFFS & HFM & 0,463 & 0,001 \\
ACAC & HFAC & 0,641 & 0,000 \\
ACAC & HFM & 0,416 & 0,005 \\
ACAC & HFFS & 0,383 & 0,006 \\
ACM & HFAC & 0,500 & 0,000 \\
ACM & HFM & 0,673 & 0,000 \\
ACM & HFFS & 0,462 & 0,001 \\
ACM & ACAC & 0,410 & 0,004 \\
ACFS & HFAC & 0,581 & 0,000 \\
ACFS & HFFS & 0,647 & 0,000 \\
ACFS & ACAC & 0,393 & 0,012 \\
ACFS & ACM & 0,280 & 0,088 \\
\hline
\end{tabular}

HFAC: Habilidades funcionais em autocuidado; HFM: Habilidades funcionais em mobilidade; HFFS: Habilidades funcionais em função social; ACAC: Assistência do cuidador em autocuidado; ACM: Assistência do cuidador em mobilidade; ACFS: Assistência do cuidador em função social

\section{DISCUSSÃO}

As crianças ribeirinhas avaliadas na presente pesquisa apresentaram desempenho funcional adequado para as áreas HFAC, HFM, HFFS, ACAC, ACFS, exceto na ACM. O ambiente não influenciou negativamente o perfil funcional das mesmas.

A pesquisa foi realizada no ambiente escolar, que é um local adequado para a prática da promoção da saúde, rastreando e problematizando os fatores determinantes das condições de saúde e doença como forma de produzir saúde. No PSE são identificados, principalmente, aqueles fatores que possam interferir no desenvolvimento integral da criança e que resultem em risco de atraso(1,2).

O desenvolvimento da criança é um processo que evolui com a idade cronológica e depende da biologia da criança e das condições ambientais, podendo levar em consideração a cultura, com seus valores e crenças, pois as restrições da tarefa, do indivíduo e do ambiente podem afetar a performance motora, comprometendo a competência motora básica ${ }^{(13,14)}$.

Em meio à natureza, as crianças podem ser surpreendidas pelas possibilidades que o espaço natural oferece e ainda favorece a estimulação dos sentidos (olfato, tato, paladar, visão e audição), o contato com os quatro elementos 
da natureza - terra, fogo, água e ar, a aproximação com a fauna e a flora local, a exploração das capacidades e habilidades motoras (subir em árvores, cavando, escalando, correndo etc.), exercícios para a autonomia e socialização, é algo positivo para a educação e o desenvolvimento das crianças ${ }^{(15)}$.

Todavia problemas relacionados às dificuldades de acesso de parte da população a serviços e bens básicos podem ser mais comprometedores ainda quando essa carência impacta os primeiros anos de vida, podendo acarretar danos ao desenvolvimento do sujeito ${ }^{(16)}$. Os achados do presente estudo corroboram a literatura, sendo analisado como um ambiente favorável para o desenvolvimento, já que nas partes de Habilidades Funcionais e Assistência do Cuidador as crianças possuem um desenvolvimento considerado adequado.

Estudo realizado em comunidade com características de infraestrutura, culturais e populacionais semelhantes à da presente pesquisa, observou que os fatores ambientais não foram barreira para o desenvolvimento neuropsicomotor das crianças ribeirinhas da comunidade amazônica Panacauera, onde a maioria das crianças não apresentava atraso no desenvolvimento ${ }^{(17)}$, corroborando os resultados da presente pesquisa. Dentre a minoria das crianças de Panacauera classificadas com atraso, o campo da linguagem foi detectado como a principal área afetada.

A família corresponde ao principal contexto de inter-relações da criança. A qualidade do ambiente familiar e das relações conduz a evolução do desenvolvimento infantil. A educação dos pais, o status socioeconômico, o tamanho da família e a interação com os irmãos são elementos principais do ambiente da criança(18). Dos cuidadores principais, $50 \%$ eram mães, corroborando com o fato da mulher ainda ser quem ocupa o papel de cuidador principal de pessoas com deficiência, algo que no Brasil é determinado culturalmente ${ }^{(19)}$.

Fatores de risco biológico (eventos pré, peri e pós-natais que resultam em danos à saúde) e ambientais, como dificuldades na aprendizagem escolar e baixo nível socioeconômico podem contribuir para o atraso no desenvolvimento motor $^{(20)}$. Nos resultados da atual pesquisa, observou-se que $62 \%$ das famílias estão classificadas na classe $D$, entretanto, a maioria das crianças apresenta funcionalidade adequada.

A funcionalidade do indivíduo é decorrente da relação entre sua condição de saúde, estruturas e funções fisiológicas, capacidade e desempenho nas atividades e participação social. É priorizada, como componente da saúde, considerando o ambiente como um facilitador ou como uma barreira para o desempenho das atividades e da participação(21).

Diante do contexto da comunidade ribeirinha, o ambiente pode ser considerado como um facilitador, devido a maioria das crianças apresentarem um desempenho funcional adequado. Um dos instrumentos mais utilizados para avaliar a funcionalidade é o PEDI, que, no modelo da Organização Mundial da Saúde contempla, juntamente com a Classificação Internacional de Funcionalidade, Incapacidade - CIF, uma análise ampliada em relação à deficiência, levando em consideração a avaliação dos níveis de atividade e participação quando o indivíduo executa uma tarefa ou ação numa situação da vida real(21,22).

O PEDI contribuiu para identificação de incapacidades individuais e das funções mais comprometidas em crianças $^{(23,24)}$. Desse modo, utilizou-se o teste com o intuito de fornecer uma descrição detalhada do desempenho da criança, para assim traçar o perfil funcional, e também por abranger a idade estabelecida pelo estudo.

Conforme os dados obtidos no presente estudo, através do escore normativo do PEDI, o desempenho funcional apresentado pela amostra em todas as áreas avaliadas pelo teste é considerado adequado para a população brasileira. O escore normativo representa o desempenho esperado de crianças brasileiras com desenvolvimento típico, visto que para cada faixa etária o escore normativo entre 30 e 70 é considerado dentro do padrão de normalidade ${ }^{(8)}$.

No resultado da área ACM que avalia a quantidade de ajuda fornecida pelo cuidador, durante a realização de alguma atividade funcional básica, $44 \%$ das crianças avaliadas na atual pesquisa apresentam um desempenho funcional atrasado. Tal dado corrobora os achados de outro estudo ${ }^{(21)}$, onde o maior índice de atraso foi na dimensão ACM. Podendo ser justificado devido às mães possuírem um número elevado de filhos e, para agilizar as atividades do dia a dia, fornecem mais ajuda do que o necessário, podendo assim explicar o percentual de atraso na área.

AAssistência do cuidador está diretamente associada ao desempenho da criança nas Habilidades funcionais de autocuidado e mobilidade ${ }^{(24)}$, sendo que o nível de independência da criança está relacionado ao seu desempenho. Dessa maneira, quanto maior o nível de desempenho nas habilidades funcionais das crianças nas áreas de autocuidado e mobilidade, menor é a quantidade de auxílio do cuidador oferecido à criança durante as atividades funcionais.

Constatou-se, também, que a redução de auxílio proporcionado pelo cuidador nas atividades de mobilidade permite maior independência das crianças nessa área, de modo que executarão com melhor desempenho as atividades funcionais de autocuidado ${ }^{(24)}$. Tal dado confirma o resultado encontrado na atual pesquisa, ressaltando ainda que, quanto maior a funcionalidade nas atividades sociais e motoras, maior a capacidade da criança na realização de tarefas do autocuidado. 
No Censo Escolar da Educação Básica de $2017^{(25)}$, as crianças com 4 a 5 anos encontram-se cursando o préescolar, e as que possuem de 6 a 9 anos, cursam o ensino fundamental (anos iniciais). Em comparação aos dados nacionais, as crianças da atual pesquisa frequentam as séries de acordo com a faixa etária estabelecida.

Um dos fatores que podem influenciar negativamente o nível educacional infantil é a baixa escolaridade das mães, pois os filhos de mulheres com baixa escolaridade tendem a entrar mais tarde na escola e a deixá-la precocemente, e esse curto tempo demonstra uma dificuldade de progredir em seus estudos ${ }^{(26)}$. Porém, nos achados do presente estudo, observou-se que mesmo as mães apresentando um nível de escolaridade baixa, isto não influenciou na série escolar de seu filho.

Como limitações do estudo tem-se a escassez de estudos que caracterizam as populações ribeirinhas, o que dificulta a fundamentação teórica de novas pesquisas e o desenvolvimento de ações para a promoção de saúde respeitando os aspectos regionais, socioeconômicos e culturais.

\section{CONCLUSÃO}

Observou-se que as crianças ribeirinhas da comunidade do Caracará, dentro da faixa etária de 5 anos a 7 anos e 6 meses, apresentam o perfil funcional adequado à sua faixa etária, segundo o Inventario de Avaliação Pediátrica de Incapacidades (PEDI), exceto para a Assistência do cuidador em mobilidade, e que o meio ambiente não influenciou negativamente suas atividades relacionadas ao autocuidado, à mobilidade e à função social.

Quanto maior o desempenho nas Habilidades funcionais, menor a quantidade de auxílio do cuidador.

\section{CONFLITOS DE INTERESSE}

Os autores declaram não haver qualquer conflito de interesses, em potencial, neste estudo.

\section{CONTRIBUIÇÕES}

Dayse Daniele de Oliveira Silva e Juliana Maciel de Queiroz contribuíram com a elaboração e delineamento do estudo; a aquisição, análise e interpretação de dados; e a redação e/ou revisão do manuscrito. Adrielly Elane Sousa Maia, Ana Paula Monteiro de Araújo, Hannah Nazareth Muribeca Athar e Paula Cristina Soares Mesquita contribuíram com a aquisição, análise e interpretação de dados; e a redação e/ou revisão do manuscrito.

\section{REFERÊNCIAS}

1. Malta DC, Reis AAC, Jaime PC, Morais OL Neto, Silva MMA, Akerman M. O SUS e a Política Nacional de Promoção da Saúde: perspectiva resultados, avanços e desafios em tempos de crise. Ciênc Saúde Colet [Internet]. 2018 [acesso em 2019 nov 5];23(6):1799-1809. doi: 10.1590/1413-81232018236.04782018

2. Ministério da Saúde (BR); Ministério da Educação (BR). Caderno do gestor do PSE. Brasília: MS; 2015.

3. Son SH, Morrison FJ. The nature and impact of changes in home learning environment on development of language and academic skills in preschool children. Dev PsycholDev Psychol [Internet]. 2010 [acesso em 2019 Ago 28];46(5):1103-18. doi: 10.1037/a0020065

4. Park MO. Comparison of motor and process skills among children with different developmental disabilities. J Phys Ther Sci [Internet]. 2015 [acesso em 2019 Ago 28];27(10):3183-84. doi:10.1589/jpts.27.3183

5. Uesugi M, Araki T, Fujii S, Itotani K, Otani Y, Seiichi T. Relationships between gross motor abilities and problematic behaviors of handicapped children in different age groups. J Phys Ther Sci [Internet]. 2014 [acesso em 2019 Set 20];26(12):1907-09. doi: 10.1589/jpts.26.1907

6. United Nations Children'S Fund. Crianças em um mundo Urbano: situação mundial da infância 2012. New York: UNICEF; 2012.

7. Teixeira SRS, Alves JM. O contexto das brincadeiras das crianças ribeirinhas da llha do Combu. Psicol Reflex Crit [Internet]. 2008 [acesso em 2019 Set 29];21(3):374-82. doi: 10.1590/S0102-79722008000300005

8. Reis DC, Araújo MEC, Santos SSL, Silva SSC, Pontes FAR. Araraiana e Combu: um estudo comparativo de dois contextos ribeirinhos amazônicos. Temas Psicol [Internet]. 2012 [acesso em 2019 Set 29];20(2):429-38. doi: 10.9788/TP2012.2-11 
9. Freitas TCB, Gabbard C, Caçola P, Montebelo MIL, Santos DCC. Family socioeconomic status and the provision of motor affordances in the home. Braz J Phys Ther [Internet]. 2013 [acesso em 2019 Set 28];17(4):319-27. doi: 10.1590/S1413-35552013005000096

10. Mancini MC. Inventário de avaliação pediátrica de incapacidade (PEDI): manual da versão brasileira adaptada. Belo Horizonte: Editora UFMG; 2005.

11. Programa das Nações Unidas para o Desenvolvimento. Ranking IDHM Municípios: 2010 [Internet]. 2010 [acesso em 2019 Set 28]. Disponível em: https://www.br.undp.org/content/brazil/pt/home/idh0/rankings/idhmmunicipios-2010.html

12. Associação Brasileira de Empresas de Pesquisa. Critério de Classificação Econômica: Brasil. São Paulo: ABEP; 2008.

13. Lima ACM. Estado nutricional e desenvolvimento motor de crianças ribeirinhas expostas ao mercúrio no Estado do Pará/ Amazônia brasileira [tese] [Internet]. Belém: Universidade Federal do Pará; 2014 [acesso em 2019 Set 29]. Disponível em: http://repositorio.ufpa.br/jspui/handle/2011/9115

14. Melo LGSC. Estudo do perfil socioeconômico, estado nutricional, nível de mercúrio e desenvolvimento motor em crianças da llha de Cotijuba, Pará / Brasil [tese] [Internet]. Vila Real: Universidade Trás-os-Montes e Alto Douro; 2016 [acesso em 2019 Out 24]. Disponível em: https://repositorio.utad.pt/bitstream/10348/6088/1/ phd_Igscmelo.pdf

15. Cocito RP. A natureza como espaço educacional: oportunidades para a infância. Colloquium Humanarum [Internet]. 2016 [acesso em 2019 Out 24];13(esp):94-100. doi: 10.5747/ch.2016.v13.nesp.000818

16. Giffoni SDA, Ribeiro MVM. Avaliação neurológica das dificuldades de aprendizagem. In: Ciasca SM, organizador. Transtornos de aprendizagem: neurociência e interdisciplinaridade. Ribeirão Preto: Book Toy; 2015. p. 161-71.

17. Pantoja APP, Souza GS, Nunes EFC, Pontes LS. Effect analysis of environmental factors on the children's Amazon community neuropsychomotor development. Rev Bras Crescimento Desenvolv Hum [Internet]. 2018 [acesso em 2019 Out 27];28(3):232-9. doi: 10.7322/jhgd.152158

18. Correa W, Minetto MDF, Crepaldi MA. Família como promotora do desenvolvimento de crianças que apresentam atrasos. Pensando Fam [Internet]. 2018 [acesso em 2019 Out 26];22(1):44-58. Disponível em: http://pepsic.bvsalud.org/pdf/penf/v22n1/v22n1a05.pdf

19. Braccialli LMP, Silva MZ, Braccialli AC, Sankako AN, Araújo RCT. Impact of school participation on quality of life of Brazilian children with cerebral palsy. Int J Disabil Hum Dev [Internet]. 2016 [acesso em 2019 Out 27];15(1):1-10. doi: 10.1515/ijdhd-2014-0024

20. Santos APM, Villaverde LN, Costa ANF, Santos MO, Gregório EC, Andreis LM, et al. Biopsychosocial factors contributing to delayed motor development in children: a longitudinal study. Rev Bras Crescimento Desenvolv Hum [Internet]. 2016 [acesso em 2019 Out 29];26(1):112-8. doi: 10.7322/jhgd.113787

21. Piexak DR, Cezar-Vaz MR, Bonow CA. Classificação Internacional de Funcionalidade, Incapacidade e Saúde: uma Análise de Conteúdo. Rev Pesqui (Univ Fed Estado Rio J) [Internet]. 2019 [acesso em 2019 Out 27];11(2):363-9. doi: 10.9789/2175-531.2019.v11i2.363-369

22. Souza MS, Braga PP. O Inventário de Avaliação Pediátrica de Incapacidade e Suas Contribuições para Estudos Brasileiros. Rev Pesqui (Univ Fed Estado Rio J) [Internet]. 2019 [acesso em 2019 Out 18];11(5):1368-75. doi: 10.9789/2175-5361.2019.v11i5.1368-1375

23. Barbosa RMF. Fatores relacionados ao desempenho funcional de crianças com idade entre cinco e sete anos [trabalho de conclusão de curso] [Internet]. Juiz de Fora: Faculdade de Fisioterapia da Universidade Federal de Juiz de Fora; 2016 [acesso em 2019 Out 26]. Disponível em: http://www.ufff.br/facfisio/files/2016/09/ Fatores-relacionados-ao-desempenho-funcional-de-crian\%C3\%A7as-com-idade-entre-cinco-e-sete-anosRaiane-Marques-Furtado-Barbosa.pdf

24. Teles FM, Resegue R, Puccini RF. Necessidades de assistência à criança com deficiência: uso do Inventário de Avaliação Pediátrica de Incapacidade. Rev Paul Pediatr [Internet]. 2016 [acesso em 2019 Out 23];34(4):447-53. doi: 10.1016/j.rppede.2016.02.015 
25. Ministério da Educação (BR). Censo Escolar da Educação Básica 2017: Caderno de instruções. Brasília: Ministério da Educação; 2017.

26. Longo FV, Vieira JM. Educação de mãe para filho: fatores associados à mobilidade educacional no Brasil. Educ Soc [Internet]. 2017 [acesso em 2019 Out 25];38(141):1051-71. doi: 10.1590/es0101-73302017162420

\section{Endereço para correspondência:}

Dayse Danielle de Oliveira Silva

Universidade do Estado do Pará - UEPA

Rodovia Augusto Montenegro, 6955/14 - 11

Bairro: Parque Verde

CEP: 66635-110 - Belém - PA - Brasil

E-mail: daysesilva@uepa.br

Como citar: Silva DDO, Maia AES, Araújo APM, Athar HNM, Mesquita PCS, Queiroz JM. Influência do ambiente sobre a funcionalidade de crianças. Rev Bras Promoç Saúde. 2019;32:9331. 